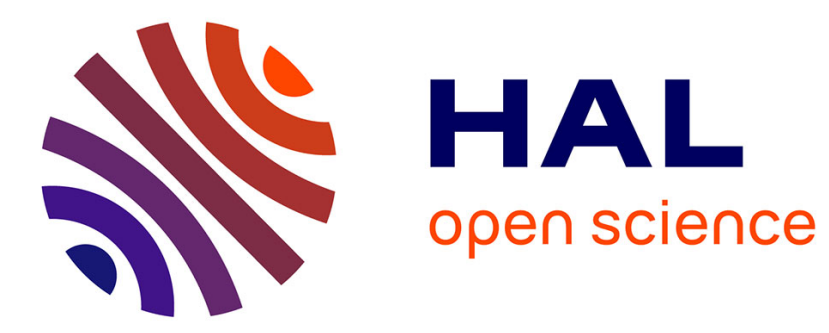

\title{
Justice et politique en Algérie 1954-1962
}

Sylvie Thénault

\section{To cite this version:}

Sylvie Thénault. Justice et politique en Algérie 1954-1962. Droit et Société, 1996, 34 (1), pp.575-587. 10.3406/dreso.1996.1385 . hal-02355882

\section{HAL Id: hal-02355882 \\ https://hal.science/hal-02355882}

Submitted on 8 Nov 2019

HAL is a multi-disciplinary open access archive for the deposit and dissemination of scientific research documents, whether they are published or not. The documents may come from teaching and research institutions in France or abroad, or from public or private research centers.
L'archive ouverte pluridisciplinaire HAL, est destinée au dépôt et à la diffusion de documents scientifiques de niveau recherche, publiés ou non, émanant des établissements d'enseignement et de recherche français ou étrangers, des laboratoires publics ou privés.

\section{(이) $\$$}

Distributed under a Creative Commons Attribution - NonCommercial - NoDerivatives| 4.0 


\section{Justice et politique en Algérie 1954-1962}

Sylvie Thénault

\section{Citer ce document / Cite this document :}

Thénault Sylvie. Justice et politique en Algérie 1954-1962. In: Droit et société, n³4, 1996. Justice et Politique (I) pp. 575-587; doi : https://doi.org/10.3406/dreso.1996.1385

https://www.persee.fr/doc/dreso_0769-3362_1996_num_34_1_1385

Fichier pdf généré le 15/05/2018 


\begin{abstract}
Justice and Politics during the Algerian War 1954-1962.

This article studies the links between justice and politics during the Algerian War (1954-1962). Algeria was considered a French " county " which explains why the war could not be declared. Algerian nationalists were seen as criminals and were put on trial. Thus, justice gave in to the political imperative, that of maintaining French sovereignty in Algeria. Indeed, governments had to use legal techniques to enforce repressive laws. Nevertheless, they neither recognized the state of war nor Algeria as a nation. Furthermore, justice had to demonstrate French sovereignty and condemn the nationalists who were contesting it. Finally, the negotiations initiated by general de Gaulle modified the judicial repression. Paradoxally enough, it became both lenient and strict at the same time given that any political negotiations imply both compromise solutions and also the taking of a hard line so as to have the upper hand.
\end{abstract}

\title{
Résumé
}

Cet article analyse les rapports entre la justice et le politique pendant la guerre d'Algérie (1954-1962), territoire alors assimilé au territoire national français. L'état de guerre ne pouvait donc être proclamé et les membres du FLN, considérés comme des criminels, furent poursuivis devant les tribunaux. La justice se soumit ainsi à l'impératif politique du maintien de la souveraineté française en Algérie : d'une part, les gouvernements usèrent des ressources de la technique juridique pour élaborer une législation d'exception qui n'entame pas le principe de l'Algérie française ; d'autre part, la justice renoua avec un double rôle historique qui était de manifester la souveraineté française sur un territoire conquis et de la sauvegarder en condamnant les nationalistes ; enfin, les négociations engagées par le général de Gaulle se répercutèrent sur la répression judiciaire qui tendit à la fois à s'adoucir et à se durcir, suivant la règle de la négociation politique qui suppose des concessions à l'adversaire et un redoublement de l'effort de guerre, dans le but d'obtenir le rapport de forces le plus favorable. 


\section{Justice et politique en Algérie 1954-1962}

\section{Sylvie Thénault*}

\section{Résumé}

Cet article analyse les rapports entre la justice et le politique pendant la guerre d'Algérie (1954-1962), territoire alors assimilé au territoire national français. L'état de guerre ne pouvait donc être proclamé et les membres du FLN, considérés comme des criminels, furent poursuivis devant les tribunaux. La justice se soumit ainsi à l'impératif politique du maintien de la souveraineté française en Algérie: d'une part, les gouvernements usèrent des ressources de la technique juridique pour élaborer une législation d'exception qui n'entame pas le principe de l'Algérie française; d'autre part, la justice renoua avec un double rôle historique qui était de manifester la souveraineté française sur un territoire conquis et de la sauvegarder en condamnant les nationalistes; enfin, les négociations engagées par le général de Gaulle se répercutèrent sur la répression judiciaire qui tendit à la fois à s'adoucir et à se durcir, suivant la règle de la négociation politique qui suppose des concessions à l'adversaire et un redoublement de l'effort de guerre, dans le but d'obtenir le rapport de forces le plus favorable.

Algérie - Décolonisation - Justice/Politique - Justice militaire - Lois d'exception - Lois de la guerre.

\section{Summary}

\section{Justice and Politics during the Algerian War 1954-1962}

This article studies the links between justice and politics during the Algerian War (1954-1962). Algeria was considered a French " county " which explains why the war could not be declared. Algerian nationalists were seen as criminals and were put on trial. Thus, justice gave in to the political imperative, that of maintaining French sovereignty in Algeria. Indeed, governments had to use legal techniques to enforce repressive laws. Nevertheless, they neither recognized the state of war nor Algeria as a nation. Furthermore, justice had to demonstrate French sovereignty and condemn the nationalists who were contesting it. Finally, the negotiations initiated

\section{L'auteur}

Agrégée d'histoire, prépare une thèse sur Les Français, l'État, la Justice pendant la guerre d'Algérie.

Parmi ses publications : - «Armée et justice pendant la guerre d'Algérie ", Le bulletin de I'IHTP, n" 63, mars 1996 ;

- «L'Algérie au cœur de l'actualité et de l'histoire", Vingtième Siècle. Revue d'histoire, n'52, octobredécembre 1996 ;

— « Les juges et la guerre d'Algérie, une nouvelle affaire Dreyfus? ", Cahiers Jean Jaurès, n 142, décembre 1996 ;

- "Assignation à résidence et justice en Algérie 1954-1962 ", Le Genre humain (à paraître).

\footnotetext{
" 17 rue du Prunet, Appartement 51, F-95100 Argenteuil.
} 
S. Thénault

Justice et politique en Algérie 1954-1962
1. Voir le texte intégral de ce manifeste dans le livre de Mohamed HARBI, Les archives de la révolution algérienne, Paris, Jeune Afrique, 1981, p. 101-103. 2. Il s'agit de Jacques Chevallier, ministre de la Défense nationale et, par ailleurs, maire d'Alger. by general de Gaulle modified the judicial repression. Paradoxally enough, it became both lenient and strict at the same time given that any political negotiations imply both compromise solutions and also the taking of a hard line so as to have the upper hand.

Algeria - Decolonization - Exceptional measures - Justice/Politics - Military justice - War's laws.

Dans la nuit du 31 octobre au $1^{\text {er }}$ novembre 1954, les nationalistes algériens lancent une offensive dont l'objectif est clairement proclamé par le tout premier manifeste du Front de libération nationale (FLN) ${ }^{1}:$ : la restauration d'un État algérien » sur un territoire conquis et occupé par la France depuis cent vingt-quatre ans, un territoire que son découpage en trois départements a soudé à la métropole. Des dizaines d'attentats commis cette nuit-là, c'est l'assassinat de l'instituteur Guy Monnerot qui émeut le plus l'opinion métropolitaine, façonnant l'image des nationalistes insurgés, présentés comme des " criminels", des " hors-la-loi ", des " rebelles".

La répression judiciaire s'opposant naturellement aux crimes et délits, cette assimilation des membres du FLN à des criminels de droit commun est à l'origine de l'intervention du juge dans une situation qui relève en réalité des lois de la guerre. Ainsi, les gouvernements auteurs des lois d'exception encadrant le conflit franco-algérien tentèrent de faire de la répression judiciaire un des piliers de la lutte contre les nationalistes. Pourtant, leur dessein, au-delà du maintien de l'ordre et de la sécurité, est politique, puisqu'il s'agit de sauvegarder l'Algérie française, jusqu'à ce que le général de Gaulle s'engage sur la voie des négociations.

Le principe de la non-reconnaissance du statut de combattant aux membres du FLN soumet donc l'activité du juge en Algérie aux impératifs de la politique algérienne des différents gouvernements. Comment les lois régissant le conflit s'accommodèrent-elles de ce principe ? Quel rôle confère-t-il à l'appareil judiciaire en Algérie ? Comment évolue-t-il avec la réorientation de la politique française en Algérie progressivement menée par le général de Gaulle?

\section{Les lois d'exception}

Dès le début du conflit, des considérations politiques s'imposent au débat juridique dont l'apparence technique est trompeuse.

C'est ainsi que, dès le 2 novembre 1954, le procureur général d'Alger, Susini, informe le ministère de la Justice que, " au cours d'une conférence qui s'est tenue dans l'après-midi du $1^{\text {cr }}$ novembre au Gouvernement général et à laquelle assistait notamment M. le ministre Chevallier ${ }^{2}$, la question s'est posée de savoir s'il convenait 
de saisir la justice militaire de l'ensemble des infractions commises par les terroristes ». La question est technique : l'article 76-2 du Code pénal permet en effet de traduire devant la juridiction militaire les auteurs de destructions d'installations pouvant servir à la défense nationale. L'atteinte à l'intégrité du territoire, si elle est connexe à d'autres crimes et délits relevant de la juridiction militaire, peut, elle aussi, lui être déférée.

À une question technique, la réponse des ministères consultés est politique. François Mitterrand, chargé de l'Intérieur, par exemple, s'oppose à la saisie systématique des tribunaux militaires, dans une lettre au garde des Sceaux le 13 novembre 1954: "Les attentats terroristes sont des crimes de droit commun. Les hommes qui commettent ces attentats contre les personnes et les biens ne sauraient en aucun cas être considérés comme ayant un caractère militaire alors que précisément la propagande antinationale s'efforce de donner ce caractère aux fellaghas. " Le principe est posé.

Pourtant, le gouvernement d'Edgar Faure, succédant à Pierre Mendès France en février 1955, franchit le pas. La loi d'état d'urgence, votée le 3 avril 1955, permet en effet à la justice militaire de revendiquer le jugement d'une large liste de crimes, allant de " tous les crimes contre la sûreté intérieure de l'État » aux "vols et recels qualifiés de crimes" en passant par "la provocation ou la participation à un attroupement criminel ", "l'association de malfaiteurs " ou encore "les incendies volontaires ». Elle peut également se saisir de la tentative ou de la complicité de ces crimes, ainsi que des délits qui leur sont connexes. La justice civile conserve cependant l'instruction de ces infractions ${ }^{3}$.

Cette disposition de compétence de la justice militaire est fondamentale dans la mesure où elle resta de mise jusqu'en 1962. En effet, après l'abrogation de l'état d'urgence en décembre 1955, les pouvoirs spéciaux, demandés et obtenus par le gouvernement de Guy Mollet le 16 mars 1956, reprennent le principe en l'élargissant. Ils autorisent les tribunaux militaires à revendiquer l'instruction de crimes dont la liste, définie par l'état d'urgence, s'enrichit de " tous crimes ou délits portant atteinte à la défense nationale " 4 . Les autorités militaires peuvent par ailleurs " ordonner la traduction directe, sans instruction préalable devant les tribunaux permanents des forces armées " des auteurs de ces crimes, pris en flagrant délit ${ }^{5}$.

Par la suite, chaque nouveau gouvernement devant demander le renouvellement des pouvoirs spéciaux, ils furent successivement accordés aux ministères conduits par Maurice Bourgès-Maunoury le 26 juillet 1957, par Félix Gaillard le 15 novembre 1957, par Pierre Pflimlin le 22 mai 1958, et par le général de Gaulle le 3 juin 1958, qui, tous, en reprirent les clauses judiciaires. Elles furent enfin prolongées sous la Ve République par le décret du 7 avril $1959^{6}$.
3. Décret 55-440 du 23 avril 1955 , adopté en vertu de la loi du 3 avril 1955.

4. Décret n'56- 268 du 17 mars 1956 , article $1^{\prime \prime}$.

5. Décret n` 56-269 du 17 mars 1956.

6. Décret n` 59-503 du 7 avril 1959. 
S. Thénault

Justice et politique en Algérie 1954-1962

7. D'après les statistiques du secrétariat général du ministère de l'Intérieur (conservées aux Archives nationales).
Comment ces gouvernements concilièrent-ils l'attribution de compétence à la justice militaire et la négation du statut de combattant aux maquisards d'Algérie, présentées comme contradictoires par François Mitterrand?

La contradiction n'est en fait pas évidente. Reconnaître le statut de combattant aux Algériens luttant pour leur indépendance impliquerait, selon les règles des conventions de Genève alors en vigueur, de les exonérer de poursuites judiciaires. Les rendre passibles des tribunaux militaires, c'est donc, au contraire, leur renier ce statut de combattant. D'ailleurs, le travail du collectif d'avocats défendant le FLN, autour de Jacques Vergès, fut précisément de contester la validité des jugements prononcés en invoquant l'application des conventions de Genève au bénéfice de leurs clients.

Malgré cela, l'implication de la justice militaire amorce l'aveu de la nature réelle des "événements d'Algérie ", pour reprendre l'euphémisme dont use la presse de l'époque. C'est en effet contraints par l'amplification de l'insurrection que les gouvernements d'Edgar Faure et de Guy Mollet, auteurs respectifs de l'état d'urgence et des pouvoirs spéciaux, sont revenus sur la décision d'écarter la justice militaire. Son intervention leur apparait comme une nécessité face aux attentats, qui, loin de régresser, persistent de novembre 1954 à avril 1955, mois durant lesquels ils se montent à près de 200, puis augmentent jusqu'à dépasser les 1000 par mois à partir de janvier 1956 ${ }^{7}$. Plus que leur nombre, c'est leur nature qui inquiète les dirigeants : les sabotages de lignes et voies de communication, les incendies criminels, les attaques à main armée et les attentats à la bombe se multiplient tandis que les voies de fait, destructions de marchandise, matériel et bétail, stagnent. Les attentats en progression sont ainsi significatifs d'une insurrection prenant l'autorité pour cible, s'éloignant du droit commun.

Prisonniers de cette situation, les gouvernements d'Edgar Faure et de Guy Mollet puisèrent alors dans les ressources de la technique juridique pour éviter que la législation d'exception s'appliquant en Algérie n'écorne l'assimilation proclamée du territoire algérien à la métropole.

Le procédé de déclaration de l'état d'urgence en est révélateur : il ne s'agit pas d'un état d'exception déclaré directement en Algérie mais d'un état d'exception introduit dans le droit français et qui peut être déclaré, par décret, dans toute zone du territoire national où l'ordre public est menacé. L'état d'urgence pourrait ainsi être déclaré en métropole si des événements semblables à ceux d'Algérie s'y produisaient. L'artifice a un double intérêt : il maintient l'assimilation territoriale de l'Algérie à la France, l'exposé des motifs de l'état d'urgence insistant sur le fait que "l'Algérie étant partie intégrante du territoire national, elle ne peut se voir dotée d'un 
régime d'exception"; ensuite, ce procédé permet de morceler le territoire algérien, niant le caractère national de l'insurrection pour la ramener au rang d'un simple "désordre " qui serait "le fait de quelques bandes organisées de hors-la-loi, numériquement peu importantes ${ }^{8}$. En effet, l'état d'urgence s'applique par étapes en Algérie: un premier décret, le 6 avril 1955, l'instaure dans les arrondissements de Batna, de Tizi-Ouzou et dans la commune de Tebessa ; puis, le 19 mai 1955, il est étendu à tout le département de Constantine, aux communes de Marnia, Sebdou, Biskra et El Oued ; enfin, le 28 août 1955, il couvre toute l'Algérie. La minimisation de la portée de l'insurrection vole donc en éclat sous la pression de la révolte des paysans dans le Nord du Constantinois, le 20 août 1955, révolte qui constituerait «le vrai début de la guerre ", selon Benjamin Stora ${ }^{9}$.

La question du statut des "hors-la-loi » est, elle, contournée par les termes du décret du 23 avril 1955 prononçant la compétence de la justice militaire. Il stipule que « les juridictions militaires peuvent être saisies, toutes les fois que les faits ont été commis postérieurement au 30 octobre 1954 dans un des arrondissements judiciaires de Batna, Guelma ou Tizi-Ouzou, des crimes désignés à l'article 2 du décret $"{ }^{10}$. La compétence de la justice militaire se définit donc en fonction de la date, du lieu et du crime commis, sans conséquence sur la qualification de leurs auteurs. Le terme même de " crime " renvoie au droit commun.

La législation des pouvoirs spéciaux, elle, ne présente pas le même risque car la répression du mouvement national algérien n'est pas son unique objet. La loi n 56-258 du 16 mars 1956, dite "des pouvoirs spéciaux", est en effet une loi " autorisant le gouvernement à mettre en œuvre en Algérie un programme d'expansion économique, de progrès social et de réforme administrative, et l'habilitant à prendre toutes mesures exceptionnelles en vue du rétablissement de l'ordre, de la protection des personnes et des biens et de la sauvegarde du territoire ». Cette fois, l'Algérie est donc distinguée de la métropole. Mais cette distinction se faisant dans l'objectif du développement de l'Algérie, elle n'en remet pas en cause le caractère français, bien au contraire. En effet, le développement promis assurerait à l'Algérie une intégration économique et sociale à l'ensemble français. Quant aux " événements », ils restent qualifiés en termes de désordre et d'insécurité, avec cependant l'introduction de la notion de "sauvegarde du territoire", avouant l'enjeu de la défense de la présence française de l'autre côté de la Méditerranée. Enfin, les décrets pris en vertu de cette loi et élargissant la compétence de la justice militaire en Algérie reprennent les termes du décret du 23 avril 1955.

La technique juridique permet donc d'aménager un système de répression qui, tout en s'opposant à un mouvement d'indépendance nationale, ne le désigne jamais comme tel et entérine le pos-
8. Exposé des motifs de la loi instituant un état d'urgence.

9. Voir sa contribution à l'ouvrage collectif La France en guerre d'Algérie, Paris, BDIC, 1992 , p. 30.

10. Article $1^{\text {"r }}$. Ces dispositions judiciaires s'étendirent ensuite à toute l'Algérie, comme l'ensemble de l'état d'urgence. 
S. Thénault

Justice et politique en Algérie 1954-1962
11. Directive (20 août 1958) du général Salan, commandant en chef des forces armées en Algérie, aux généraux commandant les corps d'armée d'Alger, d'Oran, de Constantine et les territoires de Ghardaïa, Aïn Sefra et Laghouat (conservée au Service historique de l'Armée de terre).

12. Compte rendu de cette réunion (conservé au Centre des archives contemporaines de Fontainebleau). tulat de l'Algérie française. Elle fut dans ce cas un outil d'une grande maniabilité et d'une grande efficacité pour le pouvoir politique. Les lois d'exception, ainsi rédigées, excluant toute notion de belligérance, assurèrent à la justice un rôle de première importance.

\section{Un double rôle historique}

Les lois d'exception opèrent donc un tel transfert de compétence à la justice militaire que la justice civile aurait pu se trouver dépossédée de tout rôle répressif.

En réalité, les tribunaux militaires n'étant que trois en Algérie en novembre 1954, leurs moyens matériels et humains sont limités. De plus, l'armée concentre son attention sur le verdict, étape ostensible, visible, médiatisée de toute procédure judiciaire. En effet, il entre dans le cadre de "l'action psychologique " qui occupe elle-même une place centrale au sein de la théorie de la guerre révolutionnaire développée après la guerre d'Indochine. Cette théorie veut que, face à un adversaire déployant une activité politique, l'armée sorte de son champ traditionnel d'action, celui des batailles, pour investir le champ politique et concurrencer ainsi l'adversaire sur son propre terrain. L'expression « action psychologique » recense toutes les activités menées dans le but d'influencer l'opinion algérienne dans un sens favorable au maintien de la présence française, et les jugements en font partie, comme l'explique le général Salan à ses subordonnés, en août 1958, alors qu'il leur conseille de multiplier les audiences foraines de tribunaux militaires : "... les audiences foraines multipliées, outre qu'elles accroissent le caractère d'exemplarité des sentences rendues, ont l'inestimable effet de manifester sur l'ensemble du territoire la présence et la permanence de la justice française, et de démontrer aux yeux de tous - et non seulement d'un public restreint - que la répression pénale s'exerce dans le cadre du respect scrupuleux des principes généraux en honneur dans tous les régimes démocratiques ${ }^{11}$. La partie ostensible de la procédure judiciaire présente donc un triple intérêt pour la propagande de l'armée : entretenir la peur du châtiment, rappeler la souveraineté française, répondre aux accusations de tortures et d'exécutions sommaires.

L'armée se concentrant ainsi sur le verdict, la justice civile conserve l'instruction de nombreuses procédures, que les tribunaux militaires ne lui revendiquent qu'au moment où elles sont sur le point de se clore et d'être déférées devant le tribunal. C'est ainsi que les procureurs généraux d'Alger, d'Oran et de Constantine, réunis à la Chancellerie le 3 février 1958, "précisent que presque toujours les informations sont conduites par les juges d'instruction civils, et que la revendication n'intervient qu'au terme de l'information ${ }^{12}$. De même, au terme du mois de décembre 1959, les 
plus de 3500 inculpés. La justice militaire, elle, a revendiqué les poursuites concernant seulement 195 inculpés au cours de ce même mois, soit moins de $6 \%$ du total ${ }^{13}$.

À ce rôle d'instruction s'ajoute celui du jugement des délits. Les tribunaux correctionnels jugent ainsi des centaines d'individus par mois, pour des infractions de nature politique. Au cours du mois de décembre 1959, par exemple, ces tribunaux ont jugé 317 accusés de délits tandis que les tribunaux militaires ont jugé près de 500 personnes. Les comptes rendus d'audience du tribunal correctionnel de Tiaret, rédigés par les Renseignements généraux, permettent de connaître la nature des délits qui leur sont déférés : " constitution de cellule terroriste", "aide à la rébellion" sous forme de ravitaillement, hébergement, collecte de fonds... Les condamnés sont des responsables de la structure dite "organisation politico-administrative " du FLN : commissaire politique, membres de cellules politiques du FLN, agent de liaison... ou encore les membres d'un "réseau rebelle de soutien moral et matériel "14. La justice civile assume donc un rôle de répression politique, renouant ainsi avec une tâche qui lui fut dévolue dès l'entre-deuxguerres.

Le mouvement nationaliste algérien naquit en effet au lendemain de la première guerre mondiale, avec la fondation par Messali Hadj du parti de l'Étoile nord-africaine en 1927, organisation dont les statuts incluaient la revendication de l'indépendance de l'Algérie. Dissoute en 1937, elle fut reconstituée sous le nom de Parti du peuple algérien. Messali Hadj et quatre autres dirigeants furent alors les premiers nationalistes à se voir infligés des condamnations. Arrêtés, ils écopèrent, en novembre 1937, de peines d'un ou deux ans d'emprisonnement, peine assortie, pour Messali Hadj, d'une privation des droits civiques et politiques. "Nous avions conscience de la situation, écrivit des années plus tard Messali Hadj. C'était la première fois que des nationalistes algériens étaient traduits devant un tribunal à Alger pour avoir revendiqué hautement et publiquement l'émancipation du peuple algérien et son indépendance ${ }^{15}$."

La répression judiciaire de la période de la guerre s'inscrit donc dans la continuité de la lutte contre les mouvements nationalistes qui se heurtèrent dès leurs débuts à la loi et aux tribunaux. Trente ans plus tard, d'ailleurs, de 1954 à 1962, la guerre oppose les mêmes acteurs : les fondateurs du FLN sont d'anciens militants du Parti du peuple algérien avec lequel ils ont rompu pour mettre sur pied un Comité révolutionnaire d'unité et d'action en mars 1954, embryon du FLN, et dont l'objectif était de passer à la lutte armée pour obtenir l'indépendance.

À ce premier rôle historique s'en ajoute un second, développé par le général Salan en faveur de la multiplication des audiences

13. État récapitulatif mensuel adressé par les trois procureurs généraux d'Algérie, 01/01/60 (conservé au Centre des archives contemporaines de Fontainebleau).

14. Comptes rendus d'audience du tribunal correctionnel de Tiaret, janvier-juin 1960 (conservés au Service historique de l'Armée de terre).

15. Dans ses Mémoires, Paris, Jean-Claude Lattès, 1982, p. 262. 
S. Thénault

Justice et politique en Algérie 1954-1962
16. Cité par Claude Counor, Les institutions de l'Algérie pendant la période coloniale 1830-1962, Paris, CNRS, Alger, OPU, 1987, p. 165.

17. Ibid., p. 170.

18. Justices de paix chargées des affaires civiles exclues.

19. Sud du département de Constantine. foraines de tribunaux militaires: celui de la manifestation de la "présence et de la permanence de la justice française ", c'est-à-dire de la souveraineté française sur le pays. L'argument est ancien car, déjà en 1874, le gouverneur général de l'Algérie, de Gueydon, proclamait : « La justice est un des attributs de la souveraineté, le juge musulman doit s'effacer devant le juge français; nous sommes les conquérants ${ }^{16}$. " Le déploiement de la justice française et de son activité est donc un des fondements de la colonisation d'un territoire. C'est ainsi que la période débutant en 1870 et allant jusqu'en 1962 est décrite par Claude Collot ${ }^{17}$ comme une "période d'assimilation de plus en plus poussée » du système judiciaire d'Algérie à celui de la métropole.

Dans un premier temps, les différentes institutions judiciaires de la métropole sont implantées en Algérie: cours d'assises, prud'hommes, cabinets d'avocats et d'avoués, juridictions pour mineurs... Pour ce qui est des affaires civiles cependant, les cadis, juges et notaires avant la colonisation, restent en fonction mais leur juridiction est facultative, c'est-à-dire que les justiciables peuvent également s'adresser aux juges de paix installés par la France.

La période de la guerre approfondit l'évolution sous l'influence de deux facteurs. D'une part, la contestation de la souveraineté française incite au renforcement des structures judiciaires établies en Algérie. D'autre part, l'accroissement de l'activité de l'appareil judiciaire, mobilisé face au FLN, ne peut se faire efficacement sans un investissement matériel et humain. C'est ainsi qu'en août 1955, le gouvernement d'Edgar Faure fait adopter par l'Assemblée nationale une proposition de loi déposée depuis 1951 par des députés d'Algérie. Elle crée en Algérie deux cours d'appel en sus de celle d'Alger, l'une à Oran et l'autre à Constantine. Ces cours furent installées en octobre 1956 et elles occasionnèrent l'embauche de 45 magistrats, portant à 304 l'effectif de la magistrature des cours et tribunaux en Algérie ${ }^{18}$. De même le général de Gaulle entreprit de décentraliser la justice militaire sur le sol algérien, par le décret du 7 avril 1959 qui porte à 13, au lieu de 3 auparavant, le nombre de tribunaux permanents des forces armées en Algérie. Il poursuit la politique d'assimilation avec l'application à l'Algérie de la réforme créant les tribunaux de grande instance, en février 1960, et par un projet de réforme de la justice musulmane. Ce projet, déposé en 1959 et jamais adopté, suscita la réprobation tout autant des cadis, traditionalistes, restés en place durant la colonisation, que du Gouvernement provisoire de la République algérienne, institué en septembre 1958 par le FLN.

Ce dernier et le gouvernement français se livrent une bataille de souveraineté. Le FLN a en effet interdit aux Algériens d'avoir recours aux tribunaux français et mis en place les rudiments d'une organisation judiciaire de substitution. Dans la wilaya $n^{\circ} 1^{19}$, par exemple, dès 1956, une circulaire prescrit l'organisation d'une 
assemblée du peuple de cinq membres à l'échelon du village, assemblée dont l'un des membres préside un tribunal nommé " conseil des cinq".

Même si la mise en pratique de la circulaire est aléatoire, l'important est de noter que le FLN entend bien concurrencer la France sur le terrain judiciaire. "On peut penser qu'il y a loin de l'esprit à la lettre », note ainsi le rédacteur d'un rapport sur l'organisation judiciaire du FLN en mars $1958^{20}$, mais il insiste sur "l'unité des dircctives " diffusées à ce sujet par le Comité de coordination et d'exécution du FLN, organe de direction à l'échelon national. L'interdiction du FLN ${ }^{21}$ se solde par une baisse de $60 \%$ à $90 \%$ des affaires civiles portées devant les juridictions françaises et l'auteur du rapport conclut : "... les Musulmans ne s'adressent guère à la justice française que pour faire établir les actes d'une absolue nécessité dont ils peuvent retirer un bénéfice immédiat tels que: délibération des conseils de famille, certificats de nationalité, de propriété, jugements déclaratifs de naissance, etc. »

Le développement de l'appareil judiciaire et son investissement pendant la guerre ont donc pour vocation d'affirmer la souveraineté française sur un territoire où elle est contestée et ce, en continuité avec la période d'assimilation du territoire algérien à la France.

L'implication de la justice civile dans la guerre d'Algérie renouvelle donc le double rôle que lui assigna l'histoire de la colonisation : instaurer la souveraineté française sur les territoires conquis et réprimer ceux qui la contestent.

\section{De Gaulle et les combattants}

La période de la guerre semble homogène au regard des lois d'exception et du rôle de l'appareil judiciaire. Qu'en est-il ? La justice ne subit-elle pas les contrecoups du revirement de politique accompli par le général de Gaulle à partir de septembre 1959, date à laquelle il se prononce pour l'autodétermination de l'Algérie ?

La première rupture précède en fait l'arrivée du général au pouvoir. En effet, le 13 mars 1958, le général Salan, commandant en chef des forces françaises en Algérie, prescrit d'éviter la traduction en justice des " rebelles" dits "PAM ", c'est-à-dire "pris les armes à la main » ${ }^{22}$. Il suggère la création de Centres militaires d'internés (CMI) dans lesquels les combattants seraient assignés à résidence. Cette note marque-t-elle un pas vers la reconnaissance du statut de combattant?

«Il est bien entendu que les internés ne doivent pas être considérés comme des prisonniers de guerre. Les conventions de Genève ne leur sont pas applicables ", précise lui-même le général. D'ailleurs, après avoir suggéré d'appeler les camps réservés à ces combattants Camps militaires d'internés, il se ravise, préférant le
20. Recherche sur l'organisation judiciaire rebelle en Algérie, rapport édité par le Gouvernement général de l'Algérie, mars 1958, 35 p. (conservé au Service historique de l'Armée de terre).

21. À propos de laquelle le rédacteur du rapport note : "Passer outre aux interdits du FLN, c'est pour le musulman s'exposer à perdre la vie. » 22. Note de service du 13/03/58 (conservée au Service historique de l'Armée de terre). 
S. Thénault

Justice et politique en Algérie 1954-1962
23. Statistiques du nombre des internés (conservées au Service historique de l'Armée de terre).

24. Note du sous-directeur de la direction des Affaires criminelles et des grâces,

31/12/58 (conservée au Centre des archives contemporaines de Fontainebleau).

25 . Conférence de presse du 23 octobre 1958. Texte dans les Discours et messages du général DE GAULLE, tome III : Avec le renouveau, Paris, Plon, 1970, p. 5.5 . terme de Centres militaires d'internés pour parer toute comparaison avec des camps de prisonniers de guerre. De plus, ses motivations sont purement stratégiques. En effet, il commence par constater "l'acharnement " des "rebelles acculés au combat ", qu'il présente comme le " résultat d'une préparation psychologique efficace ", les combattants de l'Armée de libération nationale (ALN) étant assurés, s'ils sont faits prisonniers, d'être exécutés après avoir été torturés ou traduits devant les tribunaux militaires qui les condamnent automatiquement à mort. "La crainte ainsi entretenue donne aux bandes un mordant qu'il importe d'entamer dans toute la mesure du possible, dans le but de réduire nos pertes. Un moyen d'y parvenir est d'accorder aux prisonniers un traitement aussi libéral que possible, et de le faire savoir. »

La note ne vaut donc pas prescription d'une reconnaissance générale du statut de combattant. D'ailleurs, tous les combattants ne sont pas exonérés de poursuites judiciaires, car " ceux qui ont commis des exactions ou qui font preuve d'un fanatisme susceptible de nuire à l'évolution favorable de l'état d'esprit d'ensemble » doivent être traduits en justice. Quant aux autres, internés, il est prévu de les soumettre dans les Centres militaires d'internés à une " action psychologique » pour leur incorporation dans les harkas, ce que les conventions de Genève interdisent.

La portée de cette mesure est d'abord limitée: les premiers Centres militaires d'internés sont ouverts en juillet 1958 et ils rassemblent à peine plus de 1000 hommes à la fin de cette année 23 . De plus, la décision de ne plus poursuivre systématiquement les "PAM " n'est entérinée par un conseil interministériel que le 18 décembre 1958, date à laquelle elle est communiquée à la Chancellerie, non informée auparavant de l'instruction du général Salan. Ce n'est finalement que le 31 décembre 1958 que le ministère de la Justice est invité à donner pour instruction aux procureurs généraux d'Algérie de ne pas poursuivre les combattants " contre lesquels on n'aurait pas à retenir d'autres infractions et ceux qui auraient agi sous la contrainte du FLN ${ }^{24}$.

L'arrivée au pouvoir du général de Gaulle marque donc la rupture réelle et cette décision judiciaire est en accord avec ses appels aux combattants de l'AlN pour qu'ils concluent une paix sans déshonneur, la célèbre "paix des braves " qu'il leur propose en octobre 1958 : "Que vienne la paix des braves [...], que ceux qui ont ouvert le feu le cessent et retournent, sans humiliation, à leur famille et à leur travail 25 ! » Il n'est cependant toujours pas question de céder sur la souveraineté française en Algérie. L'amnistie est conçue comme une mesure d'apaisement politique, nécessaire à un éventuel retour à la normale. Le résultat de cette nouvelle disposition judiciaire est que, durant les six premiers mois de 1959, sur un total de 5450 combattants faits prisonniers, $10 \%$ sont traduits en justice tandis que 50 \% sont internés. Sur les 40 \% restant, 

çaises ${ }^{26}$.

L'année 1960 est marquée par deux évolutions en apparence contradictoires. D'un côté, les premiers pourparlers s'engagent avec des représentants du FLN en juin 1960 à Melun, après que le. général de Gaulle ait employé la formule d' Algérie algérienne », pléonasme qui a cependant le mérite de s'opposer à la formule "Algérie française ». Mais, d'un autre côté, la répression judiciaire se durcit avec le décret du 12 février 1960 et l'ordonnance du 4 juin 1960. Cette double évolution s'explique par le fait que " l'Algérie algérienne " du général de Gaulle est une Algérie "liée à la France ». Il ne se résout donc pas à l'indépendance, seule solution prônée par le FLN, et doit pour ce faire négocier en position de force. " Il faut qu'il y ait le succès de nos armes. C'est seulement après des années que les Algériens auront ce qu'ils veulent ", déclare-t-il lors de son voyage en Algérie en mars 196027.

La politique judiciaire se poursuit donc dans la voie d'une répression accrue. En effet, le décret no 60-118 du 12 février 1960 instaure des procureurs militaires qui se substituent aux juges d'instruction pour rassembler les preuves nécessaires à la traduction des "rebelles " et de leurs " complices " devant les tribunaux militaires. L'enquête qu'ils mènent ne répond pas aux règles ordinaires de l'instruction et ils agissent sous l'entière autorité du commandant de zone militaire auquel ils sont hiérarchiquement soumis. À partir de cette date, le système de la revendication des procédures par la justice militaire à la justice civile n'existe plus, la justice militaire devenant compétente de plein droit pour « tous les crimes et délits contre la sûreté extérieure de l'État " et pour " tous les crimes et délits de droit commun commis en vue d'apporter une aide directe ou indirecte aux rebelles ${ }^{28}$. La justice civile est donc dessaisie à partir de juillet 1960, date d'entrée en application de ce décret. Cependant, les magistrats civils restent sur le pied de guerre : ce sont eux, en effet, qui, rappelés sous les drapeaux, remplirent les fonctions de procureurs militaires.

L'ordonnance du 4 juin ${ }^{29}$, elle, modifie la répression des crimes d'atteinte à la sûreté de l'État. Elle prévoit la répression du crime de participation à bande armée, dont certains responsables militaires pensent qu'il s'applique aux membres de l'ALN. Cette ordonnance occasionne ainsi une remise en cause des instructions antérieures qui prescrivaient de leur éviter une traduction en justice. L'hésitation ainsi née est telle que le général Crépin, commandant des forces françaises en Algérie, éprouve le besoin d'éclaircir les conditions de la traduction en justice de ceux qui sont toujours désignés comme les "PAM », à propos desquels il réitère la position du gouvernement ${ }^{30}$ : « Le gouvernement estime, tant pour des motifs d'ordre militaire (encouragement à la reddition) que pour des considérations d'ordre international (opinion étrangère, Croix

26. Fiche de renseignements de l'Armée de terre du 18 juillet 1959 (conservée au Service historique de l'Armée de terre).

27. Cité par Bernard Droz et Evelyne LEVER, dans Histoire de la guerre d'Algérie, Paris, Seuil, 1982, p. 249.

28. Article $7 \mathrm{du}$ décret.

29. Ordonnance $n^{\circ} 60-529$ du 4 juin 1960.

30. Directive particulière concernant les rebelles pris les armes à la main, 24/11/60 (conservée au Service historique de l'Armée de terre). 
S. Thénault Justice et politique en Algérie 1954-1962
31. Outre son organisation judiciaire, le FLN s'est doté de "policiers ", de " collecteurs d'impôts \%...

32. Statistiques des internés et prisonniers (conservées au Service historique de l'Armée de terre).
Rouge...), qu'il faut maintenir le système actuel. En conséquence, les rebelles pris les armes à la main (PAM) continueront en règle générale à être soustraits aux poursuites judiciaires pour être directement internés dans des camps spéciaux (CMI). »

Le principe est donc clair et maintenu. Mais son application reste problématique. "Il est impossible de donner une définition précise du PAM", indique la directive qui tente cependant de le faire en ces termes: "les combattants des bandes rebelles (djounoud) qui, en uniforme, armés, encadrés, ont mené la vie du djebel sans se compromettre dans les autres formes subversives de la rébellion ». Sont donc exclus tous ceux qui ont exercé une activité politique ou des fonctions administratives pour le compte du FLN ${ }^{31}$. Les responsables de l'ALN aux différents échelons, qui cumulent des responsabilités militaires et politiques, n'échappent donc pas à la condamnation.

De même, les combattants qui se seraient cantonnés à l'action militaire, entrant donc dans la définition précédemment donnée, doivent être poursuivis s'ils ont commis des " exactions " avec cette précision de taille : "La notion d'exactions doit être entendue dans un sens très large. " Outre " tous les actes contraires aux lois de la guerre", "toutes les attaques contre la vie et les biens des populations civiles " et les " attentats à la liberté » comme les enlèvements et les séquestrations de civils, cette notion inclut " tous les actes qui, visant principalement les forces de l'ordre, peuvent atteindre des victimes civiles " tels que le "sabotage de voies ferrées " ou la "pose d'engins explosifs " ou encore "les atteintes aux biens publics lorsqu'elles n'ont aucun rapport avec les actions de guerre".

C'est en définitive au général commandant la zone, qui détient les pouvoirs judiciaires militaires depuis le décret du 12 février 1960 , que revient la décision "d'accorder ou de refuser la qualification de PAM et par conséquent d'écarter ou de déclencher la poursuite judiciaire ", sachant qu'il "ne devra jamais perdre de vue l'esprit des directives gouvernementales ci-dessus exposées ».

Le principe de la non-reconnaissance du statut de combattant aux membres de l'ALN est donc bien remis en cause après l'arrivée au pouvoir du général de Gaulle et ce, en toute logique avec sa décision d'entamer des négociations avec le Gouvernement provisoire de la République algérienne. Mais si le revirement de principe est important, son application reste aléatoire car elle dépend du commandement. En fait, les Centres militaires d'internés ne dépassèrent pas les 5000 prisonniers de la fin de 1958 à mars 1962 alors que les effectifs de prévenus et condamnés dits " terroristes " dans les établissements pénitentiaires, sur la même période, oscillaient entre 9000 et $16000^{32}$. Les tribunaux militaires prononcèrent des peines de condamnation à mort jusqu'au 9 mars 
1962, alors que la phase finale des négociations s'était engagée à Évian.

Quant aux condamnés pour leur activité politique, qualifiée de "subversive ", ils n'ont aucun moyen d'échapper aux condamnations et ils n'obtiennent le régime politique de détention qu'en novembre 1961, soit quatre mois seulement avant la conclusion des accords d'Évian qui mettent un terme au conflit entre la France et le FLN, le 18 mars 1962.

L'impossibilité politique de reconnaître juridiquement la situation de guerre en Algérie reste donc de mise jusqu'à la fin du conflit, même lorsque le général de Gaulle décide d'ouvrir des négociations avec le gouvernement provisoire algérien. "Les CMI sont en fait des camps de prisonniers de guerre auxquels on n'a pas voulu donner cette appellation pour ne pas reconnaître de facto au FLN la qualité de belligérant », avoue une note des services de l'Armée de terre en novembre $1961^{33}$. Ce veto politique amena la justice à assumer un rôle de répression politique qui ne se désavoue pas jusqu'à la fin du conflit. La décision d'éviter les poursuites judiciaires contre les combattants stricto sensu, ne s'inscrivant pas telle quelle dans la loi, reste appliquée de manière inégale.

Comment rédgirent alors les magistrats ? Cette page d'histoire reste encore à écrire. 